\title{
Síndrome metabólica em idosos de um aglomerado urbano subnormal: prevalência e fatores associados
}

\section{Metabolic syndrome in older adults living in a subnormal urban cluster: Prevalence and associated factors}

\author{
Nathalia Barbosa de Aquino' (D), Pedro Israel Cabral de Lira² (D), Juliana Souza Oliveira ${ }^{3}$ (D), \\ Malaquias Batista Filho ${ }^{4}$ (D), Anete Rissin ${ }^{4+}$ (D), Maria de Fátima Costa Caminha ${ }^{4}$ (D), \\ Silvia Pereira da Silva de Carvalho Melo 5 (D), Alcides da Silva Diniz² (D), \\ Ilma Kruze Grande de Arruda² (i) \\ 'Programa de Pós-graduação em Gerontologia, Departamento de Medicina Social, Universidade Federal de Pernambuco (UFPE) \\ - Recife (PE), Brasil. \\ ${ }^{2}$ Departamento de Nutrição, Universidade Federal de Pernambuco (UFPE) - Recife (PE), Brasil. \\ ${ }^{3}$ Núcleo de Nutrição, Centro Acadêmico de Vitória, Universidade Federal de Pernambuco (UFPE) - Recife (PE), Brasil. \\ ${ }^{4}$ Instituto de Medicina Integral Prof. Fernando Figueira (IMIP) - Recife (PE), Brasil. \\ 5 Instituto Aggeu Magalhães, Fundação Oswaldo Cruz (Fiocruz) - Recife (PE), Brasil. \\ †'In memorian.
}

Como citar: Aquino NB, Lira PIC, Oliveira JS, Batista Filho M, Rissin A, Caminha MFC, et al. Síndrome metabólica em idosos de um aglomerado urbano subnormal: prevalência e fatores associados. Cad Saúde Colet, 2021;29(3):444-452. https://doi. org/10.1590/1414-462X202129030217

\section{Resumo}

Introdução: A síndrome metabólica (SM) é o agrupamento de fatores de risco associados à adiposidade central e à resistência à insulina. Objetivo: O objetivo desta pesquisa foi analisar a prevalência da SM e os fatores associados em idosos de uma comunidade do município de Recife, Pernambuco. Método: Estudo transversal com 166 idosos de ambos os sexos com idade igual ou superior a 60 anos, residentes na Comunidade dos Coelhos, no município de Recife. Para o diagnóstico da SM, foi utilizado o critério do NCEP-ATPIII. A análise dos dados foi efetuada com a utilização do programa estatístico SPSS para Windows, versão 13.1. Na comparação entre proporções, foram utilizados o teste de qui-quadrado de Pearson e o teste exato de Fischer. A razão de prevalência foi utilizada como medida de efeito considerando a SM como variável dependente. Resultados: A prevalência de SM encontrada foi de 38,3\%. Os fatores associados à SM após ajustes foram: a idade, na faixa etária 60-69 anos $(44,4 \%, p=0,047)$, o sexo feminino $(43,1 \%$, $p=0,056)$ e o excesso de peso $(57,3 \%, p<0,001)$. Conclusão: A SM apresentou uma alta prevalência na população idosa avaliada, sendo associada às características demográficas e ao estado nutricional. Palavras-chave: síndrome x metabólica; fatores de risco; idosos.

\begin{abstract}
Background: Metabolic syndrome (MS) is a clustering of risk factors associated with central adiposity and insulin resistance. Objective: This study aimed to analyze the prevalence of MS and associated factors in older adults living in a subnormal urban cluster located in the city of Recife, state of Pernambuco, Brazil. Method: A cross-sectional study was carried out with 166 older adults of both sexes aged $\geq 60$ years residing in the Coelhos Community in Recife. MS was diagnosed through the NCEP/ATP III criteria. Data analysis was performed using the SPSS 13.1 statistical software. The Pearson's chi-squared test and the Fisher's exact test were used to compare the proportions. Prevalence ratio was used as an effect measure considering MS as dependent variable. Results: Prevalence of MS was 38.3\%. The factors associated
\end{abstract}

Trabalho realizado na Universidade Federal de Pernambuco (UFPE) - Recife (PE), Brasil.

Correspondência: Nathalia Barbosa de Aquino. E-mail: nathalia.aquino@hotmail.com

Fonte de financiamento: nenhuma.

Conflito de interesses: nada a declarar.

Recebido em: Jun. 19, 2019. Aprovado em: Mar. 19, 2020

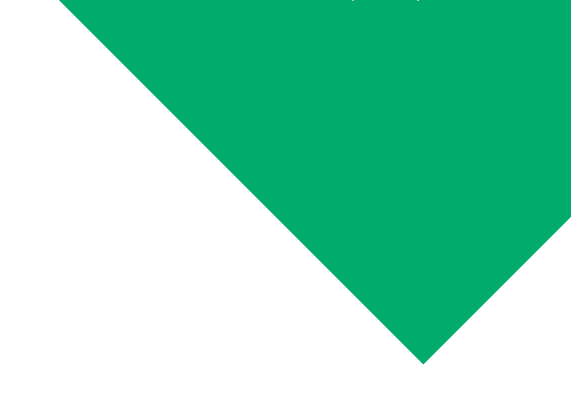


with MS after adjustments were being 60-69 years old (44.4\%, $p=0.047)$, female $(43.1 \%, p=0.056)$, and overweight $(57.3 \%, p<0.001)$. Conclusion: High prevalence of MS was observed in the older population evaluated, being associated with demographic characteristics and nutritional status. The group of older adults assessed should be provided with nutritional education and motivation to the practice of physical activities.

Keywords: metabolic syndrome $x$; risk factors; older adults.

\section{INTRODUÇÃO}

A síndrome metabólica (SM) é um conjunto de fatores de risco cardiovascular frequentemente associados à adiposidade central e à resistência à insulina ${ }^{1}$. Para o diagnóstico de SM, o Third Report of the National Cholesterol Education Program Expert Panel on Detection, Evaluation, and Treatment of High Blood Cholesterol in Adults (NCEP-ATPIII) determina a presença de três ou mais componentes no indivíduo: obesidade central, dislipidemia, hiperglicemia e hipertensão arterial sistêmica².

Além da hipertensão, hiperglicemia e dislipidemia, a SM acarreta um estado prótrombótico e pró-inflamatório'. Adicionalmente, na população idosa, a SM pode estar associada à depressão, sarcopenia, dependência funcional e comprometimento cognitivo ${ }^{3}$.

O envelhecimento populacional tem sido observado em todo o mundo ${ }^{3}$, e, concomitante a esse processo de transição demográfica, existe a transição nutricional e epidemiológica, em que há uma maior prevalência de obesidade e doenças crônicas não transmissíveis (DCNT) entre os indivíduos ${ }^{4,5}$.

Estudos têm mostrado que idosos com baixa escolaridade e em vulnerabilidade social apresentam maior probabilidade de DCNT, como diabetes e hipertensão arterial sistêmica ${ }^{6}$, sendo de suma importância investigações sobre as DCNT nessas populações, tendo em vista que, segundo o Instituto Brasileiro de Geografia e Estatística (IBGE), 26,7\% dos aglomerados urbanos subnormais se encontram na região Nordeste?.

De acordo com o IBGE, o termo "aglomerado urbano subnormal" se refere a um conjunto constituído de, pelo menos, 51 unidades habitacionais carentes, em sua maioria, de serviços públicos essenciais, validando sua exclusão social ${ }^{7}$ e necessidade de intervenções, como em um aglomerado urbano subnormal de Recife.

Por causa do rápido processo de transição demográfica, é necessária uma melhor explanação sobre os fatores associados à $\mathrm{SM}$, visando à elaboração de estratégias específicas para esse público como forma de prevenção e tratamento. Assim, o estudo teve como objetivo analisar a prevalência da SM e os fatores associados em idosos de uma comunidade em vulnerabilidade social do município de Recife, Pernambuco.

\section{MÉTODO}

Trata-se de um estudo transversal, parte integrante do estudo "Saúde, nutrição e serviços assistenciais numa população favelada do Recife: um estudo baseline", desenvolvido pelo Instituto de Medicina Integral Professor Fernando Figueira (IMIP) em parceria com o Departamento de Nutrição da Universidade Federal de Pernambuco (UFPE) e a Prefeitura Municipal do Recife.

Para o cálculo da amostra, foram tomados como referências um universo de 1.000 idosos com idade de 60 anos ou mais, uma prevalência de $53 \%$ de SM na população idosa no Brasil, um erro amostral de $7 \%$ e um nível de confiança de $95 \%$. Assim, foi obtida uma amostra de 163 idosos. A amostra final, entretanto, contou com um total de 166 indivíduos. A amostragem foi do tipo probabilística, e os idosos foram alocados por sorteio aleatório simples, sem substituição. Cabe ressaltar que no presente estudo não houve perdas amostrais.

Uma vez que o presente estudo procurou testar a associação das variáveis explanatórias com a SM, cálculos a posteriori foram realizados para estimar as diferenças do conjunto de variáveis independentes. Nessa perspectiva, foram considerados erro de 1-a de $95 \%$, poder de estudo $1-\beta$ de $80 \%$ e prevalência estimada de $65 \%$ no grupo exposto e $42 \%$ no grupo não 
exposto. Nessa condição estatística, o estudo teria um poder suficiente para detectar razões de prevalência de 1,55 ou mais como fator de associação.

A coleta de dados foi de base domiciliar, por meio de busca ativa, e ocorreu no período de junho a dezembro de 2014, na área urbana carente, localizada no município do Recife. Os dados foram coletados por pesquisadores devidamente treinados, a partir de um formulário estruturado, em que foram coletadas informações sobre condições demográficas e socioeconômicas (idade, sexo, escolaridade, classe social), informações pertinentes ao estado nutricional (peso, altura e circunferência da cintura), avaliação clínica (pressão arterial), avaliação bioquímica (glicemia de jejum, triglicerídeos e colesterol HDL) e estilo de vida (tabagismo, consumo de bebida alcoólica e atividade física). A amostra foi composta por idosos ( 60 anos ou mais), sendo identificados 478 indivíduos no total. Para compor a amostra, foram selecionados aleatoriamente 166 idosos, os quais possuíam exames bioquímicos, um pré-requisito para determinação da SM. Vale salientar que foi adotado um erro amostral de 7\% por causa das limitações de recursos financeiros para a coleta do material bioquímico.

Para a análise das variáveis sociodemográficas dos idosos, foram utilizadas as recomendações do IBGE, sendo coletadas informações concernentes ao sexo, idade e escolaridade ${ }^{8}$. Adicionalmente, os idosos foram classificados de acordo com a classe econômica a partir dos critérios estipulados pela Associação Brasileira de Empresas de Pesquisa, sendo agrupados nesta pesquisa em: classe social B1, B2, C1 e C2 e em classe social D e E'.

Para a avaliação do estado nutricional indicativo do risco para eventos cardiovasculares, o peso foi realizado em balança eletrônica com capacidade de até $200 \mathrm{~kg}$ e variação de $100 \mathrm{~g}$. A altura foi medida em duplicata, utilizando estadiômetro portátil com variação de $0,1 \mathrm{~cm}$ (admitindo-se variação máxima de 0,5 cm entre as duas medidas e calculando-se a média). O padrão de referência para a classificação das medidas de peso e altura dos idosos foi o recomendado por Lipschitz, que considera as modificações na composição corporal, próprias do envelhecimento, adotando-se os seguintes pontos de corte para a categorização dos resultados: baixo peso, IMC $<22 \mathrm{~kg} / \mathrm{m}^{2}$; eutrofia, IMC entre 22 e $27 \mathrm{~kg} / \mathrm{m}^{2}$; e excesso de peso, IMC $>27 \mathrm{~kg} / \mathrm{m}^{2}$. Na análise, foram reagrupados os pontos de corte dicotomicamente: com excesso (sobrepeso e obesidade) e sem excesso de peso (baixo peso e eutrófico) ${ }^{10}$.

Foi avaliada ainda a medida da circunferência da cintura (CC), com o uso de uma fita métrica inextensível colocada horizontalmente no ponto médio entre a borda inferior da última costela e a crista ilíaca. Para o diagnóstico da obesidade abdominal, foram adotados os pontos de corte estabelecidos pelo NCEP-APTIII, que consideram CC $\geq 102$ para homens e CC $\geq 88$ para mulheres ${ }^{2}$.

Para a aferição da pressão arterial, foi utilizado um aparelho automático com monitor MICROLIFE ${ }^{\circledR}$. Foram realizadas duas medidas no mesmo braço de cada idoso, com intervalo de 1 minuto entre elas, sendo utilizada a média dos dois valores encontrados, conforme as técnicas preconizadas pelas VI Diretrizes Brasileiras de Hipertensão (2010) ${ }^{11}$.

Para o perfil bioquímico, foram obtidos os marcadores relacionados à SM: glicemia de jejum, triglicerídeos (TG) e colesterol HDL (HDL-C). Os idosos foram orientados quanto ao jejum necessário de 12 horas para a realização do exame de sangue, com a colheita de $8 \mathrm{ml}$ de sangue venoso cubital. A glicose plasmática, o HDL-C e os TG foram analisados por meio do método enzimático colorimétrico.

Para o diagnóstico de SM, foram utilizados os critérios estabelecidos pelo NCEP-ATPIII revisado ${ }^{2}$. Segundo o NCEP-ATPIII, a SM representa a combinação de pelo menos três dos seguintes componentes: circunferência abdominal (CA), $>102$ para homens e $>88$ para mulheres; TG $>150 \mathrm{ml} / \mathrm{dL}$; baixos níveis de $\mathrm{HDL}-\mathrm{C},<40 \mathrm{ml} / \mathrm{dL}$ para homens e $<50 \mathrm{ml} / \mathrm{dL}$ para mulheres; pressão arterial elevada $\geq 130 / 85 \mathrm{mmHg}$ ou diagnóstico prévio de hipertensão; e glicemia de jejum, $\geq 100 \mathrm{ml} / \mathrm{dL}$.

Em relação às variáveis de estilo de vida (tabagismo, consumo de bebida alcoólica e atividade física), foram consideradas as seguintes categorias para o tabagismo: fumante (o idoso que relatou o hábito de fumar), ex-fumante (o idoso que relatou o hábito de fumar em algum momento da vida, porém, no momento da aplicação do questionário, não o fazia mais) e não fumante (o idoso que relatou nunca ter fumado). Para a variável consumo de bebida 
alcoólica, foi avaliado o consumo de bebidas alcoólicas nos 30 dias anteriores à aplicação do questionário. Para efeito de análise, foram formadas as categorias de resposta: sim ou não ${ }^{12}$.

Para a análise da atividade física, foi utilizado o International Physical Activity Questionnaire (IPAQ, 2001), em sua versão curta. Foram considerados suficientemente ativos os idosos que apresentaram atividade moderada, vigorosa ou caminhada por tempo $\geq 150$ minutos/semana, e insuficientemente ativos ou sedentários os idosos com atividade moderada, vigorosa ou caminhada por tempo $<150$ minutos $/$ semana $^{13}$.

Os dados foram analisados por meio do software SPSS Statistics, versão 13.1. As variáveis contínuas foram testadas quanto à normalidade da distribuição, pelo teste de KolmogorovSmirnov. As variáveis com distribuição normal foram expressas na forma de média e desviopadrão. As variáveis com distribuição não gaussiana foram apresentadas sob a forma de medianas e dos respectivos intervalos interquartílicos. Na comparação entre proporções, foi empregado o teste qui-quadrado de Pearson, quando necessário. A razão de prevalência foi utilizada como medida de efeito, considerando a SM como variável dependente. Para todas as análises, foi considerado o nível de significância de 5\%.

O projeto foi submetido ao Comitê de Ética em Pesquisa em Seres Humanos do IMIP (Projeto de Pesquisa no 3201-12), em atendimento às normas regulamentares de pesquisas envolvendo seres humanos (Resolução n 466/12, do Conselho Nacional de Saúde), e aprovado por ele.

\section{RESULTADOS}

A amostra foi composta por 166 idosos, dos quais a maioria (69,3\%) era do sexo feminino, com uma maior predominância na faixa etária entre 60 e 69 anos (63,9\%). Ainda, 61,2\% eram analfabetos ou possuíam apenas o ensino fundamental incompleto, bem como $54,8 \%$ pertenciam às classes sociais mais empobrecidas. O excesso de peso foi observado em $54,4 \%$ dos idosos, $76 \%$ afirmaram nunca ter fumado, $78,8 \%$ não fizeram uso de bebida alcoólica e $63,2 \%$ foram considerados insuficientemente ativos (Tabela 1).

Foi observada uma prevalência de 38,3\% (IC95\% = 30,7-46,5) para SM entre os idosos da Comunidade dos Coelhos. A prevalência da SM foi estaticamente maior entre a faixa etária de 60 a 69 anos (44,4\%), assim como entre o sexo feminino (43,1\%), e essa associação se situou no limiar da significância estatística, com 0,047 e 0,056, respectivamente. O estado nutricional se mostrou associado ao desfecho $(p<0,001)$. Além disso, mais da metade $(57,3 \%)$ dos idosos com excesso de peso apresentou SM, mas não houve diferenças significativas com relação às outras variáveis (Tabela 2).

\section{DISCUSSÃO}

A alta prevalência encontrada nesta pesquisa (38,3\%) mostra o quão o grupo de idosos (60 anos ou mais) está susceptível às DCNT, especialmente por causa das mudanças fisiológicas próprias do processo de envelhecimento. Estudo feito no Piauí também encontrou uma prevalência semelhante $(38,64 \%)^{14}$, enquanto resultados superiores foram descritos em pesquisas realizadas por Saad et al. ${ }^{15}$ no Rio de Janeiro e por Santos et al. ${ }^{16}$ no estado do Pará, que encontraram uma prevalência de $45,2 \%$, e $51,6 \%$, respectivamente, enfatizando a necessidade de elaboração de políticas de saúde específicas para o público idoso.

As prevalências diferentes encontradas nos estudos de Saad et al. ${ }^{15}$ e Santos et al. ${ }^{16}$ ressaltam a necessidade de pesquisas no país que considerem as singularidades regionais e étnicas de cada território brasileiro, haja vista os diferentes valores de prevalência para SM em idosos, sobretudo para que se encontre um critério diagnóstico mais sensível e específico para esse grupo populacional.

No presente estudo, utilizando-se do critério diagnóstico do NCEP-ATPIII revisado², foi verificado que a prevalência da SM foi maior entre o sexo feminino. Estudos realizados por Rocha et al. ${ }^{17} \mathrm{e}$ Vieira et al. ${ }^{18}$ também encontraram resultados similares, e algumas considerações poderiam explicar esse resultado: a composição da amostra ter mais mulheres, seguindo o processo de feminização da velhice ${ }^{19}$, e também pelo fato de que a senescência leva à 
Tabela 1. Caracterização da população segundo as variáveis sociodemográficas, estilo de vida e estado nutricional de idosos residentes em um aglomerado urbano subnormal, Recife, Pernambuco, 2014

\begin{tabular}{|c|c|c|c|}
\hline Variáveis & $\mathbf{N}$ & $\%$ & $I_{95 \%}$ \\
\hline \multicolumn{4}{|l|}{ Sexo } \\
\hline Masculino & 51 & 30,7 & $24,2-38,1$ \\
\hline Feminino & 115 & 69,3 & $61,8-75,7$ \\
\hline \multicolumn{4}{|l|}{ Idade } \\
\hline $60-69$ anos & 106 & 63,9 & $56,3-70,7$ \\
\hline$\geq 70$ anos & 60 & 36,1 & $29,2-43,6$ \\
\hline \multicolumn{4}{|l|}{ Escolaridade } \\
\hline Analfabeto/ fundamental I incompleto & 101 & 61,2 & $53,6-68,3$ \\
\hline Fundamental I completo/ fundamental II incompleto & 40 & 24,2 & $18,3-31,2$ \\
\hline Fundamental 2/ médio/superior & 24 & 14,5 & $9,9-20,7$ \\
\hline \multicolumn{4}{|l|}{ Classe social } \\
\hline $\mathrm{B} 1$ e $\mathrm{B} 2 / \mathrm{C} 1$ e $\mathrm{C} 2$ & 75 & 45,2 & $37,8-52,7$ \\
\hline De $E$ & 91 & 54,8 & $47,2-62,2$ \\
\hline \multicolumn{4}{|l|}{ Estado nutricional } \\
\hline Sem excesso de peso & 67 & 45,6 & $37,7-53,6$ \\
\hline Com excesso de peso & 80 & 54,4 & $46,4-62,3$ \\
\hline \multicolumn{4}{|l|}{ Tabagismo } \\
\hline Fumante/ ex-fumante & 35 & 24,0 & $17,7-31,5$ \\
\hline Nunca fumou & 111 & 76,0 & $68,4-82,2$ \\
\hline \multicolumn{4}{|l|}{ Consumo de bebida alcoólica } \\
\hline Sim & 31 & 21,2 & $15,3-28,5$ \\
\hline Não & 115 & 78,8 & $71,4-84,6$ \\
\hline \multicolumn{4}{|l|}{ Atividade física* } \\
\hline Insuficientemente ativo & 72 & 63,2 & $54,0-71,4$ \\
\hline Suficientemente ativo & 42 & 36,8 & $28,5-45,9$ \\
\hline
\end{tabular}

*Suficientemente ativos: indivíduos que realizam atividade moderada, vigorosa ou caminhada por tempo $\geq 150$ minutos/semana. Insuficientemente ativos ou sedentários: indivíduos com atividade moderada, vigorosa ou caminhada $<150$ minutos/semana.

redistribuição da gordura corporal e a um maior acúmulo da gordura visceral, especialmente em mulheres idosas ${ }^{20}$.

A maior predominância da SM na faixa etária de 60 a 69 anos é um dado de certa forma esperado, uma vez que a hipertensão arterial sistêmica é um dos componentes da SM, e um dos fatores de risco não modificável para hipertensão é a idade. Com o envelhecimento, ocorrem alterações vasculares, em que há um enrijecimento vascular e disfunção endotelial. Esse enrijecimento da parede arterial é resultado do rompimento de suas fibras elásticas com substituição por tecido colágeno, originando um aumento da pressão ${ }^{21}$. Estudo feito por Falsarella et al..$^{22}$ relatou a variação da composição corporal durante o processo de 
Tabela 2. Razão de prevalência de síndrome metabólica de acordo com as variáveis sociodemográficas, medidas de estilo de vida e estado nutricional de idosos residentes em um aglomerado urbano subnormal, Recife, Pernambuco, 2014

\begin{tabular}{|c|c|c|c|c|c|}
\hline \multirow[t]{2}{*}{ Variáveis } & \multirow{2}{*}{$\begin{array}{l}\text { Total } \\
\mathbf{N}(\%)\end{array}$} & \multirow{2}{*}{$\begin{array}{c}\begin{array}{c}\text { Síndrome } \\
\text { metabólica }\end{array} \\
\mathrm{N}(\%)\end{array}$} & \multirow[t]{2}{*}{$\mathbf{R P}$} & \multirow{2}{*}{$I C_{95 \%}$} & \multirow[t]{2}{*}{ p-valor* } \\
\hline & & & & & \\
\hline \multicolumn{6}{|l|}{ Idade } \\
\hline $60-69$ anos & $90(63,8)$ & $40(44,4)$ & 1 & & \\
\hline$\geq 70$ anos & $51(36,2)$ & $14(27,5)$ & 0,62 & $0,37-1,02$ & 0,047 \\
\hline \multicolumn{6}{|l|}{ Sexo } \\
\hline Masculino & $39(27,7)$ & $10(25,6)$ & 1 & & \\
\hline Feminino & $102(72,3)$ & $44(43,1)$ & 1,68 & $0,94-3,00$ & 0,056 \\
\hline \multicolumn{6}{|l|}{ Escolaridade } \\
\hline $\begin{array}{l}\text { Ensino fundamental II/ ensino médio/ ensino } \\
\text { superior }\end{array}$ & $18(12,8)$ & $7(38,9)$ & 1 & & \\
\hline $\begin{array}{l}\text { Ensino fundamental I completo/ ensino } \\
\text { fundamental II incompleto }\end{array}$ & $34(24,1)$ & $12(35,3)$ & 0,91 & $0,43-1,90$ & 0,80 \\
\hline Analfabeto/ ensino fundamental I incompleto & $89(63,1)$ & $35(39,3)$ & 1,01 & $0,54-1,91$ & 0,97 \\
\hline \multicolumn{6}{|l|}{ Classe social } \\
\hline $\mathrm{B} 1$ e $\mathrm{B} 2 / \mathrm{C} 1$ e $\mathrm{C} 2$ & $61(43,3)$ & $23(37,7)$ & 1 & & \\
\hline De E & $80(56,7)$ & $31(38,8)$ & 1,03 & $0,67-1,57$ & 0,90 \\
\hline \multicolumn{6}{|l|}{ Estado nutricional } \\
\hline Sem excesso & $65(46,4)$ & $10(15,4)$ & 1 & & \\
\hline Com excesso & $75(53,6)$ & $43(57,3)$ & 3,73 & $2,04-6,81$ & $<0,001$ \\
\hline \multicolumn{6}{|l|}{ Tabagismo } \\
\hline Nunca fumou & $104(78,8)$ & $41(39,4)$ & 1 & & \\
\hline Fumante/ ex-fumante & $28(21,2)$ & $10(35,7)$ & 0,91 & $0,52-1,57$ & 0,72 \\
\hline \multicolumn{6}{|l|}{ Consumo de bebida alcoólica } \\
\hline Não & $106(80,3)$ & $43(40,6)$ & 1 & & \\
\hline Sim & $26(19,7)$ & $8(30,8)$ & 0,76 & $0,41-1,41$ & 0,36 \\
\hline \multicolumn{6}{|l|}{ Atividade física } \\
\hline Suficientemente ativos & $40(37,7)$ & $14(35,0)$ & 1 & & \\
\hline Insuficientemente ativos & $66(62,3)$ & $25(37,9)$ & 1,08 & $0,64-1,83$ & 0,77 \\
\hline
\end{tabular}

RP: razão de prevalência; IC: intervalo de confiança

*Teste de qui-quadrado de Pearson.

envelhecimento, mostrando que há uma redução da massa magra a partir da quinta década de vida, enquanto o tecido adiposo aumenta gradativamente em ambos os sexos até a sétima década de vida. Após essa década, a tendência é que diminua a circunferência da cintura e, consequentemente, o risco de desenvolver $\mathrm{SM}$, já que, segundo o consenso brasileiro de $\mathrm{SM}^{1}$, a síndrome está relacionada com a deposição central de gordura. 
Dessa forma, estudos têm apresentado uma menor prevalência entre os idosos com idade igual ou superior a 70 anos $^{23,24}$. Entretanto, pesquisa produzida por Cabrera e Jacob ${ }^{25}$ mostra que a diminuição de obesidade entre a faixa etária de 80 anos ou mais pode sugerir a influência da obesidade e das patologias a ela associadas como fatores que poderiam estar colaborando para a maior mortalidade dos idosos obesos com 80 anos. Ainda observou que não houve diferença de prevalência de obesos entre os idosos de 60 a 69 anos e de 70 a 79 anos, mostrando que a prevalência de obesidade não se apresenta de forma homogênea nas diferentes faixas etárias.

Com relação ao estilo de vida, pesquisas mostram a relação entre tabagismo e etilismo com a hipertensão arterial sistêmica, justificando a frequência encontrada neste estudo. Ademais, a Sociedade Brasileira de Cardiologia recomenda a cessação do tabagismo e a moderação no consumo de álcool para o melhor controle da pressão arterial ${ }^{11}$.

Assim como estudo feito por Scherer e Vieira ${ }^{26}$, a maior parte dos idosos da comunidade apresentava baixa escolaridade e baixa renda. Segundo Feliciano ${ }^{27}$, a organização social do começo do século bloqueou o acesso à escola aos mais pobres e às mulheres. $\mathrm{O}$ baixo nível escolar compromete o acesso de qualidade aos programas de educação em saúde que propiciam a adesão de comportamentos saudáveis ${ }^{28}$. Além disso, estudo produzido por Campos e Barbieri ${ }^{29}$ relatou que os idosos também migram para as cidades grandes com o objetivo de ter a companhia dos filhos e/ou parentes e acesso a serviços de saúde especializados, evidenciando os aglomerados urbanos em condições precárias de vida.

Nesta pesquisa, cerca de metade da população dos idosos estudados apresentou excesso de peso. Estudo realizado por Mello et al..$^{30}$ encontrou resultado semelhante, mostrando que a epidemia de obesidade no Brasil atinge, inclusive, a população geriátrica e bolsões de pobreza. Estudos nacionais realizados com idosos, como o inquérito sobre Saúde, Bem-estar e Envelhecimento (SABE) ${ }^{31}$ e o estudo de coorte Saúde e Envelhecimento do Bambuí32, também trouxeram dados preocupantes com relação à elevada prevalência do excesso de peso na população idosa. Esses resultados podem estar relacionados com questões fisiológicas próprias do processo da senescência, em que há alterações fisiológicas, anatômicas e metabólicas que afetam o estado nutricional do idoso, com aumento da gordura corporal total e redução do tecido muscular ${ }^{33}$.

Esse resultado também pode estar associado à baixa prática de atividade física e ao processo de globalização alimentar, em que há uma padronização da alimentação, com aumento da ingestão de alimentos processados e ultraprocessados, ricos em açúcares, gorduras e sódio ${ }^{34}$. É importante enfatizar ainda que o excesso de peso é o precursor das alterações dos demais componentes da $\mathrm{SM}^{16}$.

Ademais, foi visto que apenas $36,8 \%$ dos idosos praticavam atividade física. Sousa et al. ${ }^{35}$ encontraram um percentual de $25,4 \%$ e Ribeiro et al. ${ }^{36}$ apresentaram uma prevalência de $29,9 \%$ de idosos suficientemente ativos, mostrando que a prática de atividade física entre a população idosa ainda é baixa, podendo ser inferido que uma menor renda familiar está relacionada à dificuldade no acesso a espaços de atividade física, bem como disponibilidade de tempo livre para a prática de atividade física. É possível também ter relação com o fato de que populações com baixo nível de escolaridade possuem ligação com altos índices de criminalidade ${ }^{37}$, o que gera receio de praticar atividades físicas em espaços abertos, por deixar os indivíduos mais expostos à violência.

Por fim, mesmo com uma amostra pequena, o estudo apresentou resultados significativos com relação ao estado nutricional, sexo e idade, o que enaltece os dados encontrados no estudo realizado, retratando de fato a realidade. Entretanto, são necessários mais estudos com amostras mais robustas para determinar, de forma mais clara, a associação da SM com outras variáveis.

A SM se mostrou associada ao sexo, idade e estado nutricional. A associação da SM com o estado nutricional sugere uma maior atenção com relação às medidas preventivas, de acordo com a singularidade do grupo estudado, bem como respeitando as particularidades de gênero, tendo em vista a maior prevalência no sexo feminino.

A relação de escassas avaliações no Brasil sobre a ocorrência de DCNT e elevada prevalência de SM encontrada entre os idosos da comunidade carente estudada mostra, em caráter preliminar, a necessidade da elaboração de políticas de saúde específicas para o público 
geriátrico, principalmente nas comunidades em vulnerabilidade social, tendo em vista que a SM já é uma realidade de muitos idosos.

Com relação aos componentes da SM, foi analisado que a hipertensão arterial foi maior entre os idosos com excesso de peso e que a HDL-c alterada foi maior entre os idosos insuficientemente ativos, sendo imprescindível, novamente, o estímulo às atividades físicas.

Os resultados desta pesquisa apontam ainda que os idosos residentes em uma área de exclusão social apresentam baixo nível de escolaridade, podendo influenciar diretamente a baixa receptividade às atividades de educação em saúde e, portanto, uma baixa adesão a tratamentos e medidas preventivas, resultando em uma alta frequência de obesidade e SM e uma baixa prática de atividade física.

\section{REFERÊNCIAS}

1. Sociedade Brasileira de Cardiologia. I Diretriz Brasileira de Diagnóstico e Tratamento da Síndrome Metabólica. Arq Bras Cardiol. 2005;84(Supl. 1):3-28. http://dx.doi.org/10.1590/S0066-782X2005000700001.

2. Grundy SM, Cleeman JI, Daniels SR, Donato KA, Eckel RH, Franklin BA, et al. Diagnosis and management of the metabolic syndrome: an American Heart Association/National Heart, Lung, and Blood Institute Scientific Statement. Circulation. 2005;112(17):2735-52. http://dx.doi.org/10.1161/CIRCULATIONAHA.105.169404. PMid:16157765.

3. Roriz-Cruz M, Rosset I, Wada T, Sakagami T, Ishine M, Roriz-Filho JS, et al. Stroke-independent association between metabolic syndrome and functional dependence, depression, and low quality of life in elderly community-dwelling Brazilian people. J Am Geriatr Soc. 2007;55(3):374-82. http://dx.doi.org/10.1111/ j.1532-5415.2007.01068.x. PMid:17341239.

4. World Health Organization. Relatório mundial de envelhecimento e saúde. Geneva: WHO; 2015.

5. Batista M Fo, Rissin A. A transição nutricional no Brasil: tendências regionais e temporais. Cad Saude Publica. 2003;19(Supl. 1):S181-91. http://dx.doi.org/10.1590/S0102-311X2003000700019. PMid:12886448.

6. Vitoi NC, Fogal AS, Nascimento CM, Franceschini SCC, Ribeiro AQ. Prevalência e fatores associados ao diabetes em idosos no município de Viçosa, Minas Gerais. Rev Bras Epidemiol. 2015;18(4):953-65. http:// dx.doi.org/10.1590/1980-5497201500040022. PMid:26982308.

7. Instituto Brasileiro de Geografia e Estatística. Aglomerados subnormais primeiros resultados: censo demográfico. Rio de Janeiro: IBGE; 2010. 259 p.

8. Instituto Brasileiro de Geografia e Estatística. Household Budget Survey 2002-2003: analysis of household food availability and nutritional status in Brazil. Rio de Janeiro: IBGE; 2004.

9. Associação Brasileira de Empresas de Pesquisa. Dados com base no Levantamento Sócio Econômico 2010 - IBOPE [Internet]. 2012 [citado em 2019 jun 19]. Disponível em: http://www.abep.org/

10. Lipschitz DA. Screening for nutritional status in the elderly. Prim Care. 1994;21(1):55-67. http://dx.doi. org/10.1016/S0095-4543(21)00452-8. PMid:8197257.

11. Sociedade Brasileira de Cardiologia. Hipertensão e nefrologia. VI Diretrizes Brasileiras de Hipertensão Arterial. Arq Bras Cardiol. 2010;95:1-51.

12. Pinho CPS, Diniz ADS, Arruda IKGD, Lira PIC, Cabral PC, Siqueira LAS, et al. Consumo de alimentos protetores e preditores do risco cardiovascular em adultos do estado de Pernambuco. Rev Nutr. 2012;25(3):341-51 http://dx.doi.org/10.1590/S1415-52732012000300004.

13. Matsudo S, Araújo T, Matsudo V, Andrade D, Andrade E, Oliveira LC, et al. Questionário Internacional de Atividade Física (Ipaq): Estudo de validade e reprodutibilidade no Brasil. Atividade Física \& Saúde. 2001;6(2):5-18.

14. Silva J Fo, Freire JAP, Frota KDMG, Silva AJD Jr. Fatores de risco cardiovascular e prevalência de síndrome metabólica em idosos. Rev Bras Promoç Saúde. 2014;27(4):447-84. http://dx.doi. org/10.5020/18061230.2014.p477.

15. Saad MAN, Cardoso GP, Martins WA, Velarde LG, Cruz RA Fo. Prevalência de síndrome metabólica em idosos e concordância entre quatro critérios diagnósticos. Arq Bras Cardiol. 2014;102(3):263-9. PMid:24676226.

16. Santos PCM, Ferreira ALL, Mori RMSC. Frequência da Síndrome Metabólica em idosos cadastrados no Programa Saúde do Idoso de uma Unidade Municipal de Saúde de Belém-PA. Rev Assoc Bras NutriçãoRASBRAN. 2017;8(1):75-81. 
17. Rocha FL, Melo RLP, Menezes TN. Fatores associados à síndrome metabólica em idosos do interior do Nordeste brasileiro. Rev Bras Geriatr Gerontol. 2016;19(6):978-86. http://dx.doi.org/10.1590/198122562016019.160046.

18. Vieira EC, Peixoto MDRG, Silveira EAD. Prevalence and factors associated with Metabolic Syndrome in elderly users of the Unified Health System. Rev Bras Epidemiol. 2014;17(4):805-17. http://dx.doi. org/10.1590/1809-4503201400040001. PMid:25388482.

19. Almeida AV, Mafra SCT, Silva EP, Kanso S. A Feminização da Velhice: em foco as características socioeconômicas, pessoais e familiares das idosas e o risco social. Textos \& Contextos. 2015;14(1):115-31. http://dx.doi.org/10.15448/1677-9509.2015.1.19830.

20. Figueiredo JAD No, Figuerêdo ED, Barbosa JB, Barbosa FDF, Costa GRC, Nina VJDS, et al. Síndrome metabólica e menopausa: estudo transversal em ambulatório de ginecologia. Arq Bras Cardiol. 2010;95(3):339-45. http://dx.doi.org/10.1590/S0066-782X2010005000094. PMid:20658092.

21. David CND. Associação de sobrepeso e obesidade abdominal com mortalidade geral e cardiovascular em idosos de 80 anos ou mais: um estudo de coorte [dissertação]. Porto Alegre: Universidade Federal do Rio Grande do Sul; 2015.

22. Falsarella GR, Gasparotto LPR, Coimbra IB, Coimbra AMV. Envelhecimento e os fenótipos da composição corporal. Rev Kairós. 2014;17(2):57-77.

23. Mahabaleshwarkar R, Taylor YJ, Spencer MD, Mohanan S. Prevalence of metabolic syndrome in a large integrated health care system in North Carolina. N C Med J. 2016;77(3):168-74. http://dx.doi.org/10.18043/ ncm.77.3.168. PMid:27154881.

24. Closs VE, Feoli AMP, Schwanke CHA. Síndrome metabólica em idosos da atenção terciária em Porto Alegre, Rio Grande do Sul: associação com o Índice de Alimentação Saudável. Sci Med. 2016;26(3):6. http://dx.doi. org/10.15448/1980-6108.2016.3.23422.

25. Cabrera MA, Jacob W Fo. Obesidade em idosos: prevalência, distribuição e associação com hábitos e co-morbidades. Arq Bras Endocrinol Metabol. 2001;45(5):494-501. http://dx.doi.org/10.1590/S000427302001000500014.

26. Scherer F, Vieira JLDC. Estado nutricional e sua associação com risco cardiovascular e síndrome metabólica em idosos. Rev Nutr. 2010;23(3):347-55. http://dx.doi.org/10.1590/S1415-52732010000300003.

27. Feliciano AB, Moraes SA, Freitas ICM. O perfil do idoso de baixa renda no Município de São Carlos, São Paulo, Brasil: um estudo epidemiológico. Cad Saude Publica. 2004;20(6):1575-85. http://dx.doi.org/10.1590/ S0102-311X2004000600015. PMid:15608859.

28. Dias JAA, Oliveira RF, Santos MLC, Nery PIG. Desafios vivenciados por clientes com hipertensão arterial para adesão ao tratamento dietético. Rev Enferm UFPE on line. 2016;10(10):3825-32.

29. Campos MB, Barbieri AF. Considerações teóricas sobre as migrações de idosos. R Bras Est Pop. 2013;30:6984. http://dx.doi.org/10.1590/S0102-30982013000400005.

30. Mello APA, Belo LADO, Pontes AEB, Pagotto V, Nakatani AYK, Martins KA. Estudo de base populacional sobre excesso de peso e diabetes mellitus em idosos na região metropolitana de Goiânia, Goiás. Geriatr Gerontol Aging. 2016;10(3):151-7. http://dx.doi.org/10.5327/Z2447-211520161600001.

31. Lebrão ML, Laurenti R. Saúde, bem-estar e envelhecimento: o estudo SABE no Município de São Paulo. Rev Bras Epidemiol. 2005;8(2):127-41. http://dx.doi.org/10.1590/S1415-790X2005000200005.

32. Giacomin KC, Uchôa E, Firmo JO, Lima-Costa MF. Projeto Bambuí: um estudo de base populacional da prevalência e dos fatores associados à necessidade de cuidador entre idosos. Cad Saude Publica. 2005;21(1):80-91. http://dx.doi.org/10.1590/S0102-311X2005000100010. PMid:15692641.

33. Ciosak SI, Braz E, Costa MFBNA, Nakano NGR, Rodrigues J, Alencar RA, et al. Senescência e senilidade: novo paradigma na atenção básica de saúde. Rev Esc Enferm USP. 2011;45(Spec No 2):1763-8. http:// dx.doi.org/10.1590/S0080-62342011000800022. PMid:22569669.

34. Diez Garcia RW. Reflexos da globalização na cultura alimentar: considerações sobre as mudanças na alimentação urbana. Rev Nutr. 2003;16(4):483-92. http://dx.doi.org/10.1590/S1415-52732003000400011.

35. Sousa CAD, César CLG, Barros MBDA, Carandina L, Goldbaum M, Marchioni DML, et al. Prevalência de atividade física no lazer e fatores associados: estudo de base populacional em São Paulo, Brasil, 20082009. Cad Saude Publica. 2013;29(2):270-82. http://dx.doi.org/10.1590/S0102-311X2013000200014.

36. Ribeiro AQ, Salgado SML, Gomes IS, Fogal AS, Martinho KO, Almeida LFF, et al. Prevalência e fatores associados à inatividade física em idosos: um estudo de base populacional. Rev Bras Geriatr Gerontol. 2016;19(3):483-93. http://dx.doi.org/10.1590/1809-98232016019.150047.

37. Becker KL, Kassouf AL. Uma análise do efeito dos gastos públicos em educação sobre a criminalidade no Brasil. Econ Soc. 2017;26(1):215-42. http://dx.doi.org/10.1590/1982-3533.2017v26n1art8. 